\title{
Massive secondary postpartum hemorrhage caused by pseudoaneurysm of uterine artery, treated with uterine artery embolization - An uncommon case report
}

\author{
loannis Kokolakis, Fanis Makrigiannakis, Afroditi Ziogou, Antonios Makrigiannakis
}

University Hospital of Heraklion, Department of Obstetrics and Gynecology

Corresponding Author

loannis Kokolakis, email: kokolakisioannis@gmail.com

\section{Abstract}

Introduction: Uterine artery pseudoaneurysm is an uncommon cause of secondary postpartum hemorrhage that can be fatal. Material \& Methods: We present a case of a 42-year-old woman who developed massive secondary postpartum hemorrhage 17 days after c-section and was diagnosed to have pseudoaneurysm from the left uterine artery on computed tomography scan. After blood transfusion she got hemodynamically stable and selective transcatheter arterial embolization was performed. Results: The pseudoaneurysm was successfully obliterated. Conclusions: Angiographic embolization is a safe and competent method for managing postpartum hemorrhage caused by pseudoaneurysm in hemodynamically stable patients. However, uterine artery ligation may be the preferred method for hemodynamically unstable patients when fertility preservation is strongly desired. In a woman with unexplained vaginal bleeding after c-section, pseudoaneurysm should be considered in the differential diagnosis of secondary postpartum hemorrhage. Angiographic embolization should be examined as a treatment method before turning to surgery, in suitable selected cases.

Key words: Postpartum hemorrhage, embolization, pseudoaneurysm

\section{Introduction}

Secondary postpartum hemorrhage is described as extreme bleeding starting within 24 hours following delivery up to 6 weeks postpartum and usually appearing between 8 and 14 days postpartum. In such cases, physicians should firstly exclude the presence of retained products of conception, of partial or incomplete involution of the placental bed and the possibility of endometritis. Rare cases of secondary postpartum hemorrhage include the presence of pseudoaneurysms of the uterine artery, arteriovenous malformations, and choriocarcinoma ${ }^{1}$.

Pseudoaneurysm is the result of extra-luminal 
collection of blood. This is usually encircled from the adventitia of the vessel or in rarer cases which can be fatal from perivascular soft tissue. The absence of a three-layer arterial wall lining in the pseudoaneurysm is distinctive and helps establish the differential diagnosis from a true aneurysm. When the supplying blood originates from the uterine cavity it can cause persistend and severe secondary hemorrhage. Pseudoaneurysms may be asymptomatic, may thrombose, or can result in distal embolization that is usually manifested as painful swelling of the distal part that is involved. The risk of rupture is equivalent to the size and intramural pressure ${ }^{2}$.

Uterine artery pseudoaneurysm is responsible for almost $3 \%$ of cases of postpartum hemorrhage and can be fatal in certain cases when the defect that supplies the pseudoaneurysm is large. The occurrence of an arterial pseudoaneurysm of uterine artery is an uncommon but described complication of pelvic surgery, vascular trauma during c-section or after uterine curettage. The most frequent cause of uterine artery pseudoaneurysm is cesarean delivery.

Diagnosis of pseudoaneurysm of uterine artery is established with the use Doppler sonography and arteriography. Transcatheter uterine artery embolization (UAE) is a highly successful technique for treating both gynecologic and obstetric hemorrhage, including pseudoaneurysms ${ }^{2,3}$.

\section{Case report}

A 42 years old, gravida 1 woman was transferred to our hospital 17 days post c - section with symptoms of massive vaginal bleeding. She had undergone an elective c-section for suspected macrosomia and because of patient's desire. She was obviously asymptomatic for 15 days post operation. Later, she developed massive vaginal bleeding.

On abdominal examination, the c-section scar appeared to be normal and no any other abnormality was recognized. She was extremely heavy bleeding per vaginum. The uterus was bulky and the cervical os was closed. Her hemoglobin (Hb) level was $4.1 \mathrm{~g} /$ $\mathrm{dl}$ and hematocrit (Ht) $12.6 \%$. Her blood pressure was 92/59 mmHg and Heart Rate was 129 bpm. Transvaginal ultrasonography with color Doppler revealed obviously a postpartum uterus with no apparent abnormality. So, there was no evidence of remaining placental tissue in the uterine cavity \& endometrial thickness was only $7 \mathrm{~mm}$. Then Computed tomography (CT) angiogram was performed and diagnosis of pseudoaneurysm from the left uterine artery was established. She then was stabilized with crystalloids, four units packed red blood cells (RBC), and started on broad spectrum antibiotics immediately.

After blood transfusion she got hemodynamically stable. To preserve the fertility in this young woman a transcatheter arterial embolization of this pseudoaneurysm was scheduled. She underwent digital subtraction angiography. Selective left uterine artery angiogram revealed a pseudoaneurysm from the terminal part of the left uterine artery (Figure 1). Left uterine artery was carefully embolized with:1) mixture of gelfoam and contrast media \& 2) followed by 1 stainless steel coil $4 \mathrm{~mm}$ in diameter. A post embolization angiography was done to confirm the complete occlusion of the vessel. The pseudoaneurysm was successfully obliterated (Figure 2). The patient preserved her uterus.

\section{Discussion}

Postpartum hemorrhage is considered a major cause of maternal morbidity and mortality, particularly in developing countries ${ }^{2}$. Accurate and timely diagnosis of the underlying cause is of paramount importance to help stabilize the patient and avoid severe morbidity. Vascular causes of delayed postpartum hemorrhage (other than iatrogenic injury) are rare. Avoidance of unnecessary curettage in cases that do not present with retained products of con- 


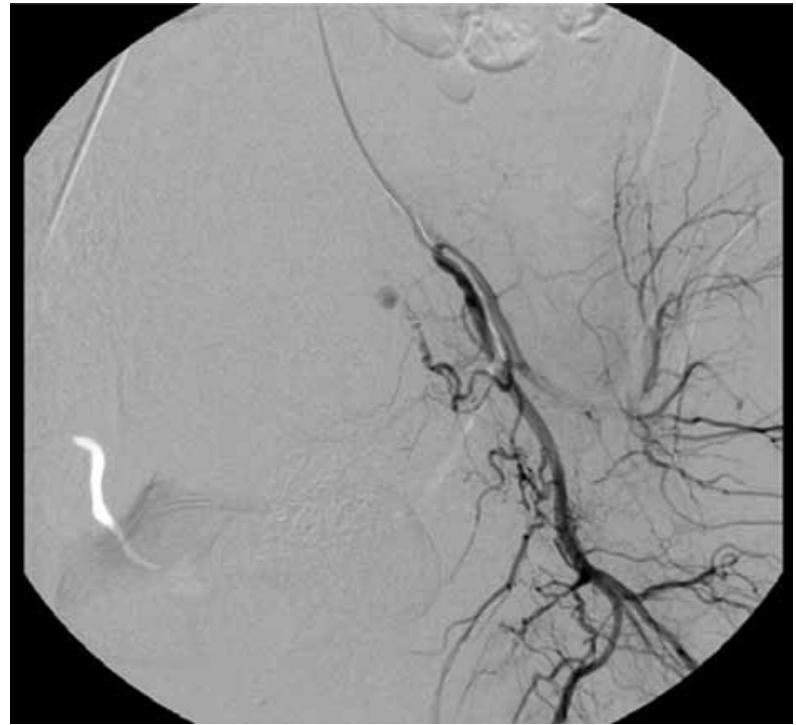

Figure 1. Selective left uterine artery angiography demonstrates a pseudoaneurysm from the terminal part of the left uterine artery (arrow)

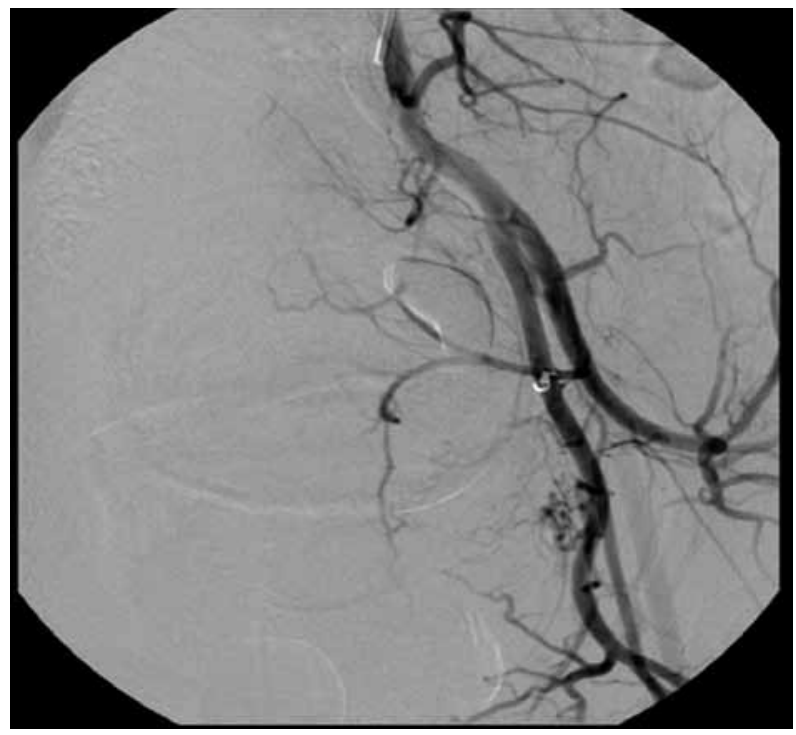

Figure 2. Post-embolization angiography of the left uterine. There is no evidence of a pseudoaneurysm

ception is prudent in order to avoid fatal blood loss. With the establishment of latest imaging techniques, the diagnosis of uterine artery pseudoaneurysm has become easy. The diagnosis of a pseudoaneurysm can be made by color Doppler ultrasonography, computed tomography (CT), magnetic resonance imaging, and angiography. Angiography, however is the gold standard method in diagnosis of uterine artery pseudoaneurysm ${ }^{4}$.

Typically, development of pseudoaneurysms is a complication of vascular injury resulting from trauma, inflammation, or iatrogenic causes such as surgical procedures. Cesarean section is the most prevalent cause (it counts for approximately $47 \%$ of cases $^{3,5}$. The cause of arterial injury, resulting in the formation of the uterine artery pseudoaneurysm in our case, is unknown. The uterine incision for the Cesarean section as well as the sutures that were used during its closure could both explain its presence. The addition of a second layer of hemostatic sutures to control the bleeding at the angles of the uterine incision could also lead to the formation of the pseudoaneurysm ${ }^{4,6}$. The interval between pelvic surgery and beginning of symptoms ranges between 1 week and 3 months, but delayed cases observed as late as 2 years later have been also reported. In a review by Isono et al, the mean timeframe between cesarean section and presentation with symptoms was 14.5 days. In our case hemorrhage developed 17 days post c-section ${ }^{7,8}$.

Uterine artery pseudoaneurysm accounts for nearly $3 \%$ of cases of postpartum hemorrhage. Currently, the incidence of uterine artery pseudoaneurysms is increasing. This is most likely because of an increase in cesarean section rates and higher detection rates cause of technological advances ${ }^{9}$.

In 1979, Brown, et al described the first successful case of selective arterial embolization for treatment an extra-uterine pelvic hematoma after three failed surgical attempts. From that time, arterial embolization has been used extensively to control postpartum bleeding with the success rate following embolization around 97\%, which is high ${ }^{9}$. Young Ho Choi, studied the effectiveness of uterine artery embolization 
and reported $90 \%$ overall success rate with $10 \%$ complications, which suggests that selective angiographic embolization could be considered a first-line diagnostic and treatment method for uterine artery pseudoaneurysm ${ }^{10}$. Surgical intervention such as a hysterectomy or an internal iliac arterial ligation is preferred in hemodynamically unstable women. The described success rates for internal iliac artery ligation differ from $42 \%-100 \%$ because of the large pelvic collateral circulation ${ }^{11}$.

\section{Conclusion}

In a patient with abnormal vaginal bleeding after c-section, the presence of pseudoaneurysm should be examined as one of the possible causes of secondary postpartum hemorrhage. Pseudoaneurysm is a probably fatal complication ${ }^{2}$. Angiographic embolization is a safe, practical and applicable method for managing postpartum hemorrhage due to pseudoaneurysm in hemodynamically stable patients. However, for hemodynamically unstable patients uterine artery ligation should be preferred when we desire fertility preservation.

Angiographic embolization is preferred cause of: 1) decreased morbidity, 2) the capability to localize the bleeding site 3) to provide a more distal occlusion than surgical ligation and 4) the preservation of future fertility in contrast to hysterectomy. Although data are insufficient, bilateral uterine artery embolization for obstetric hemorrhage turns out to not decrease subsequent fertility and is more successful when compared to unilateral embolization.

Thus, angiographic embolization is a safe, practical and efficient method for managing postpartum hemorrhage caused by pseudoaneurysm in hemodynamically stable patients.

\section{References}

1. Weeks A. The prevention and treatment of postpartum haemorrhage: what do we know, and where do we go to next? BJOG. 2015 Jan;122(2):202-10.

2. Subramaniam S, Nadarajan C, Aziz ME. Role of Uterine Artery Embolization in Pseudoaneurysm of Uterine Artery: A Rare Cause of Secondary Postpartum Hemorrhage. Cureus. 2018 Feb 23;10(2):e2220.

3. Ugwumadu L, Hayes K, Belli AM, Heenan S, Loftus I. Uterine artery pseudoaneurysm requiring embolization in pregnancy: a case report and review of the literature. CVIR Endovasc. 2018;1(1):31.

4. Dasari P, Maurya DK, Mascarenhas M. Uterine artery pseudoaneurysm: a rare cause of secondary postpartum haemorrhage following caesarean section. BMJ Case Rep. 2011 Mar 25;2011.

5. Moatti Z, Nisner T, Saini A, Karoshi M. Delayed postpartum haemorrhage secondary to a ruptured uterine artery pseudo-aneurysm, successfully treated by transarterial embolisation. BMJ Case Rep. 2011 Dec 1;2011.

6. Chummun K, Kroon N, Flannelly G, Brophy D. Severe Postcoital Bleeding From a Uterine Artery Pseudoaneurysm 4 Months After Cesarean Delivery. Obstet Gynecol. 2015 Sep;126(3):638-41.

7. Johannesson L, Mason J, Oehler MK. Uterine artery pseudoaneurysm bleeding one decade after caesarean section: A case report.J Obstet Gynaecol Res. 2017 May;43(5):939-942.

8. Isono $\mathrm{W}$, Tsutsumi R, Wada-Hiraike O, Fujimoto A, Osuga Y, Yano T. Uterine Artery Pseudoaneurysm after Cesarean Section: Case Report and Literature Review. J Minim Invasive Gynecol. 2010 Nov-Dec;17(6):687-91.

9. Nanjudan P.,Rohilla M, Raveendran A, Jain V, Khandelwal N. Pseudoaneurysm of uterine artery: a rare cause of secondary postpartum hemorrhage, managed with uterine artery embolisation. J Clin Imaging Sci. 2011;1:14.

10. Choi YH, Baba Y, Ikeda S, et al. Efficacy of uterine artery embolization for patients with postpartum hemorrhage. Open J Radiol. 2013;3:5. 
11. Singh Abha et al. Ligating Internal Iliac Artery:

Received 30-04-20

Success beyond Hesitation. J Obstet Gynaecol In-

Revised 17-05-20

dia. 2016 Oct; 66(Suppl 1): 235-241.

Accepted 18-05-20 